\title{
Cosmic magnetic field studies with the SKA and their analysis
}

\author{
J. Geisbuesch ${ }^{1}$ and P. Alexander ${ }^{1,2} \star$
}

\author{
1 Astrophysics Group, Cavendish Laboratory, Madingley Road, Cambridge, CB3 OHE, United Kingdom \\ 2 Kavli Institute for Cosmology Cambridge, Madingley Road, Cambridge, CB3 OHA, United Kingdom
}

\begin{abstract}
We discuss simulations of the polarised sky in the frequency range of the SKA mid-band. From our polarisation simulations, which are built on semi-empirical extra-galactic radio continuum simulations, empirically as well as numerically derived intrinsic source population polarisation properties and radiation propagation from the emitting source along the lineof-sight to the observer, we obtain synthetic polarisation observations assuming SKA telescope specifications as defined by the most recent reference design study. For the purpose of radiation transfer, we utilise a halo model approach to obtain the spatial distribution of the Faraday depth induced by galaxies, groups and clusters. Our modelling includes local halo physical as well as global cosmological aspects. By applying Bayesian analysis methods based on Markov Chain Monte Carlo and Nested Sampling techniques to spectro-polarimetric data sets we investigate how well cosmic magnetic fields can be studied and their properties inferred from prospective polarisation data.
\end{abstract}

\section{Introduction}

The evolution of cosmic structure is mainly governed by gravity. However, there are indications that on small scales magnetism may play a significant role. While gravity has been well studied, the origin and evolution of magnetic fields in the Universe is still very little understood, even though radio observations of synchrotron emission indicate that major parts of the Universe are pervaded by them. Commonly, it is assumed that seed magnetic fields are amplified by dynamo mechanisms. These seed fields, which can later be fostered by dynamo actions, might be generated by Weibel instabilities or the Biermann battery mechanism. A powerful tool for studying magnetic fields in a variety of environments is to use Faraday rotation against background and embedded polarised sources. New and up-coming instruments, such as the SKA and its pathfinders, will allow for the first time to perform detailed studies of the magnetic Universe via Faraday rotation measure (RM) techniques. In the presented work, we investigate which insights can be gained on magnetic field evolution from observations by these instruments on the basis of cosmological simulations of the distribution of polarised emitters and the magneto-ionic medium in large scale structures (LSS), such as clusters, groups and galaxies.

\section{Simulations of the polarised sky}

Very little is known about faint radio source populations, especially in respect to their polarisation properties. To model such populations realistically we follow the approach as presented in Wilman et al. 2008 and also use the publicly available SKA Design Study (SKADS) Sky Simulations $\left(\mathrm{S}^{3} ; \mathrm{s}\right.$-cubed . physics.ox.ac.uk). The catalogue is derived from semiempirical simulations of different populations of radio contin-

\footnotetext{
^ This work was supported by the European Commission Framework Program 6, Project SKADS, Square Kilometre Array Design Studies (SKADS), contract no 011938.
}

uum sources and extends down to faint flux limits in order to allow observation simulations of high-sensitive future radio facil ities, such as the SKA and the Low Frequency Array (LOFAR). The source populations included in the catalogue are radio-loud (FRI and FRII) and radio-quiet AGNs as well as radio emitting starburst and normal disk galaxies. These populations are of utmost importance, if not constituting a complete list of radio emitting source types in the relevant range of observing frequencies and flux regimes. The luminosity function corresponding to each class of sources is derived empirically from available observations and can be updated as soon as new observational insights become available. The fits are extrapolated to low luminosities and assume redshift evolutions to assure the simulations are complete down to the faint flux limits, which are reachable by future radio telescopes.

To determine the degree of polarisation of individual sources, we employ existing polarisation data. For example, our modelling makes use of polarisation statistics obtained from the NVSS catalogue (Condon et al. 1998). Studies of polarised radio sources and their polarisation degrees were carried out using this data set by Tucci et al. 2003 and Mesa et al. 2002. Their results indicate that for radio sources with deep spectra, the mean fractional polarisation increases with decreasing flux density. Recently, Taylor et al. 2007 confirmed this finding and studied the polarised source population to even fainter fluxes. We model source polarisation based on these studies. For example, we assume a median fractional polarisation of $1.77 \%$ for NVSS sources with Stokes I fluxes from 100-200 mJy and use a luminosity dependent modelling for the polarisation of radio-loud AGNs. Another way to obtain polarisation degrees of sources is to astrophysically model them. This can be done in an analytic or numerical fashion. The polarisation distribution of a population can then be obtained from these models by Monte Carlo simulations. In this way for instance we derived polarisation degree statistics for normal and starburst galaxies. Figure 1 shows a patch of a realisation of a polarisation sky simulation. 

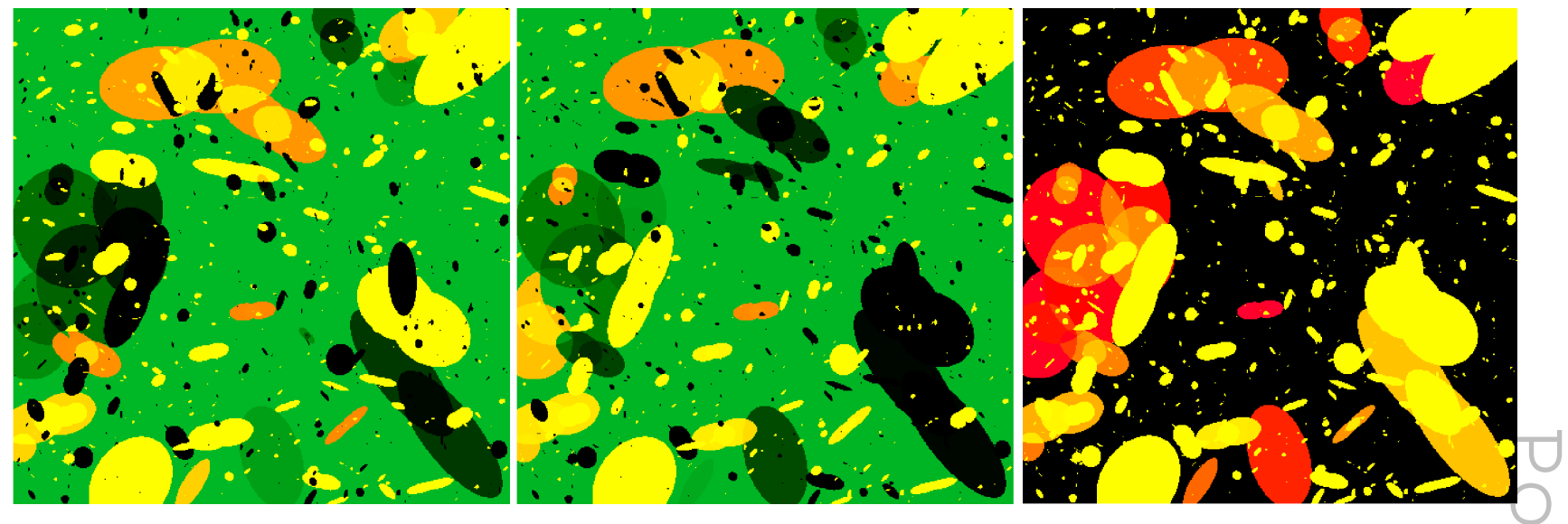

Fig. 1: A realisation of the polarised sky at $1 \mathrm{GHz}$ is shown. For illustration purposes only a small fraction of the sky ( $\sim 0.001$ square degree $) \Omega$ is displayed in total polarisation $P=\sqrt{Q^{2}+U^{2}}$ (right panel) and Stokes parameters $Q$ (left panel) and $U$ (mid panel). The plots are obtained using zscaled histogram equalisation.

Apart from polarised sources, Faraday screens in between the source and the observer affect the polarisation signal. Most dominant screens are constituted by LSS in the Universe with large range coherent magnetic fields, i.e. galaxy clusters. Rotation measures (RMs) in galaxy clusters have been found to exceed occasionally $1000 \mathrm{rad} / \mathrm{m}^{2}$ in cluster core regions. However, also galaxies and thus the Milky Way as well as ionospheric disturbances in the Earth's upper atmosphere contribute.

Our Faraday sky simulations are built on a halo model approach (see e.g. Krause et al. 2009; Geisbuesch et al. 2008, 2009a). Here, we employ N-body simulations as well as Lagrangian perturbation theory in order to derive the spatial distribution of halos of different masses within redshift ranges. Further halo properties, which are not directly obtained from these simulations, are derived via scaling relations. For example, scalings between the halo mean magnetic field amplitudes and halo masses are assumed. Further assumptions about their redshift evolution, which are physically and/or observationally motivated, are made. Magnetic field structures within clusters are generated by assuming a power spectrum for the spatial distribution of the magnetic field vector components. Thereby our modelling ensures that the vector field is divergence free. Furthermore, we scale the cluster magnetic field strength by a profile so that it decreases radially outwards. Note that the applied magnetic field modelling is in good agreement with observations (see e.g. Murgia et al. 2004; Govoni et al. 2006). The electron gas density profile is derived from the distribution of dark matter in the halo, which is modelled by a NFW profile, by assuming hydrostatic equilibrium. Figure 2 shows a simulated Faraday sky patch obtained by the outlined modelling recipe.

In previous work (Krause et al. 2009), we modelled the cluster RM distribution by a smooth $\beta$-profile. For visualisation purposes we apply a similar model in Figure 3 in order to demonstrate the effect the magneto-ionic cluster atmosphere has on the observed polarisation signal from background and embedded sources. The resulting map has been convolved with a beam of 1 arcsec FWHM. Note that sources behind the clus-

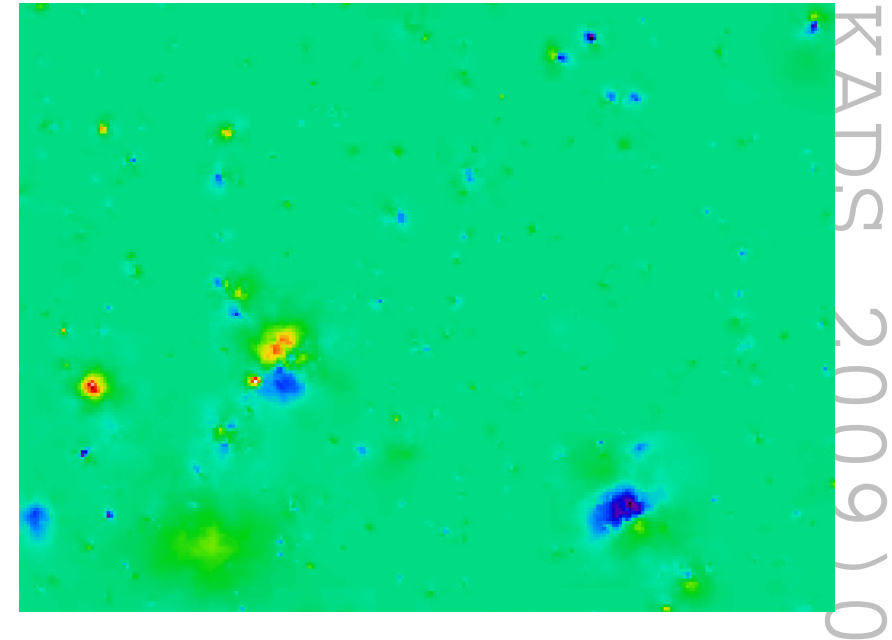

Fig. 2: Sky section of an RM realisation for a concordance $\Lambda \mathrm{CDM}$ model.

ter centre region are clearly depolarised due to beam depolarisation.

\section{Synthetic observations}

The polarisation sky simulations as described in the previous section present a tool that is very useful for determining how instrumental properties can affect radio spectro-polarimetric research. The potential of telescope designs to achieve certain research goals can be verified by observation simulations of synthetic polarised sky realisations. Studies of how alterations of the telescope design or different assumptions about the sky signal change results are cost-effectively realisable. As an example, we show here how instrumental properties can limit scientific return by the means of a resolution study. For this purpose we assume the resolution properties of three different generations of radio interferometers. The noise level in the simulations corresponds to a $100 \mathrm{hrs}$ SKA observation and is consistent with the SKA design specifications as given in Schilizzi 

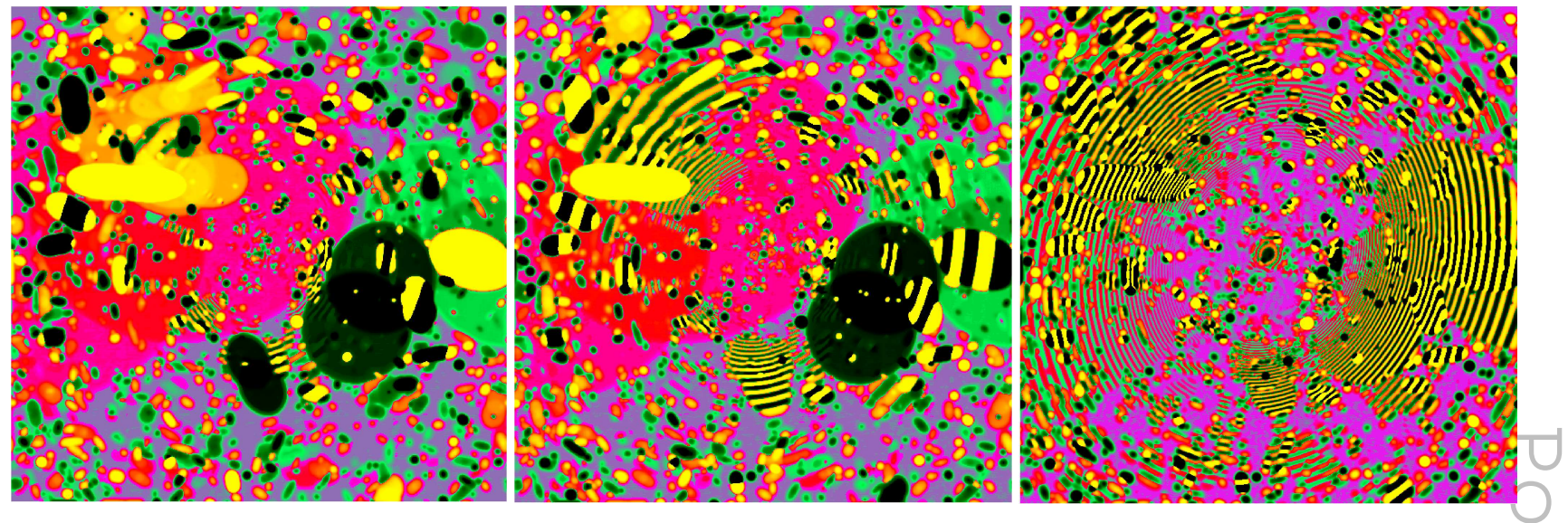

Fig. 3: Realisation of Stokes $Q$ for a similar patch as shown in Figure 1. This time a cluster Faraday screen has been placed at different redshifts $\Omega$ (left panel: $z=0.5$, mid panel: $z=0.3$ and right panel: $z=0.01$ ). Some of the sources are in the foreground of the cluster and their polarised emission is therefore not affected by the cluster's magneto-ionic medium. For the purpose of clearly visualising the effect of the Faraday rotation of the polarisation angle, we used a smooth profile to model the cluster Faraday depth. The maps have been convolved with a beam of FWHMof 1 arcsec.

et al. 2007. As it is well visible in Figure 4, the sky appears quite different at different resolutions. This is mainly due to the fact that at high resolution the sources and their polarisation structures are resolved. The polarised signal from extragalactic sources often originates from extended lobes rather than compact cores (see also Figure 1). Thus, in the case that angular resolution is very high, the extended polarised emission is in many cases hidden in the noise or dependent on the telescope design can be even resolved out. Conversely, if the angular resolution is too low, many sources suffer beam depolarization by unresolved field structures.

\section{Polarisation data analysis}

We employ Bayesian methods to analyse source polarisation data, namely Markov Chain Monte Carlo (MCMC) and Nested Sampling (NS). Both methods yield comparable results with NS being often computationally less expensive. We derive accuracies on RMs dependent on the instrument design, in particular the instrument bandwidth, and the signal-to-noise ratio of the source detection. A detailed discussion of the applied methods and results can be found in Geisbuesch \& Alexander 2009b. The implemented algorithms are applied to the spectropolarimetric data of polarised point-like sources (linear Stokes parameters: $Q$ and $U$ ). Sources can be detected in the first place by standard source detection techniques as applied in a wide spectrum of astronomical areas, e.g. source detection in multi-frequency microwave and submillimetre observations. Different to most current implementations of RM-synthesis, our algorithm performs its analysis in frequency rather than RM space. This saves the computationally costly Fourier transform and subsequent RM-cleaning. Figure 5 shows results of the relative RM accuracy for a range of RM values in dependence on the over the band integrated signal-to-noise ratio of the source polarisation signal. Note that even at relatively low signal-to-noise ratios of $S N R \sim 3$ the screen RM value can be
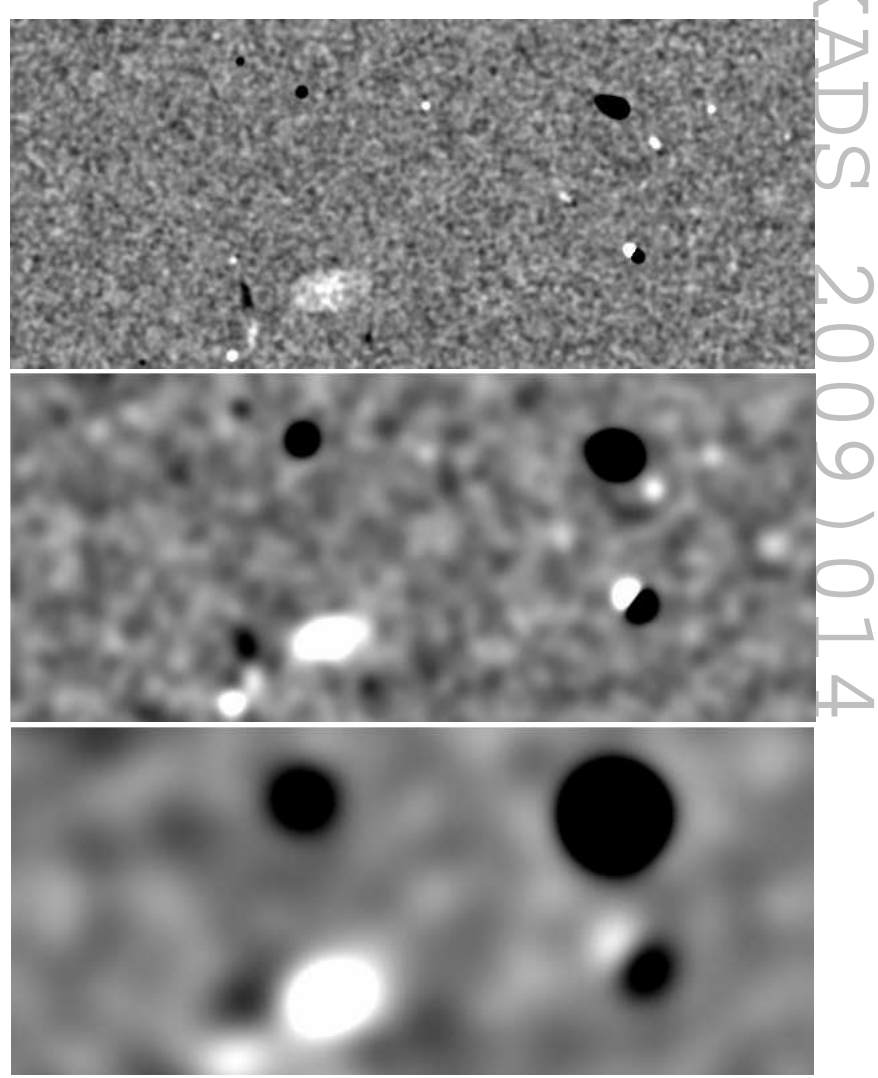

Fig. 4: A resolution study: the assumed instrument beam is varied while the noise level is kept constant. The panels show the same patch at a resolution of $1 \operatorname{arcsec}$ (top panel), $15 \operatorname{arcsec}$ (mid panel) and 40 arcsec (bottom panel). The polarised sky simulation includes polarised sources and Faraday screens. Stokes Q is shown.

recovered with sufficient accuracy. Above this signal-to-noise ratio the RM accuracy agrees well with theoretical predictions. 

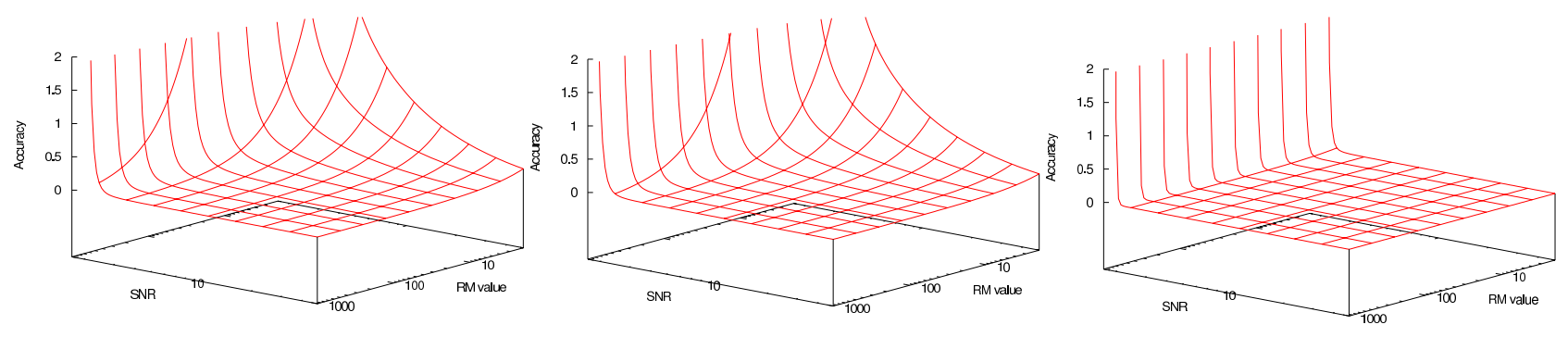

Fig. 5: Dependence of the relative accuracy of the RM value on the signal-to-noise ratio and the actual RM value of the screen for the two main pathfinder experiments of the SKA, ASKAP (left panel) and MeerKAT (mid panel), and for the SKA itself (right panel).

\section{SKA RM survey missions}

Based on the simulations and the data analysis we can estimate the density of RM grids for different observing strategies of the SKA and determine to which redshifts cosmic magnetism can be studied. In particular, our goal is to determine how the magnetic field strength in the intracluster medium evolves with redshift. In order to make a reliable measurement of the cluster magnetic field, we assume that more than 10 RMs through a cluster must be detected. If the redshift evolution of the magnetic field can be described by a redshift-dependent term of $(1+z)^{\alpha}$ with $\alpha>0$, several ten thousand clusters have to be detected to enable us to discover an evolution as slow as $\alpha=0.3$ at $10 \sigma$ (see e.g. Alexander et al. 2007).

In the following, we investigate 'cluster detection' for two different surveying strategies, a wide and a deep one. In the case of a wide field survey covering the full southern hemisphere in a year of observing, an RM grid density of $\sim 4000-5000$ sources per square degree can be achieved considering the latest SKA design concepts. The requirement of $10 \mathrm{RMs}$ for a 'cluster detection' is then achievable for more than 100,000 groups and clusters, which are mostly located at redshifts $z \lesssim 0.5$, in a concordance model. However, these clusters require SunyaevZel'dovich (SZ) and/or X-ray follow-up to confirm their location and to obtain estimates of the electron gas densities along the lines-of-sight. The selection of such follow-up limits the number of clusters, for which all required information is available, to $\sim 70,000$ for deep or respectively $\sim 12,000 \mathrm{ob}-$ jects for shallow follow-up. Therefore, stacking objects of similar masses within a well-defined redshift range will increase the usable number of clusters and will help to constrain cluster magnetic field properties.

A deep SKA polarimetry survey covering 40 square degrees with $100 \mathrm{hrs}$ integration time per pointing will allow us to study magnetic fields of groups and clusters of galaxies and their evolution up to high redshifts $(z \sim 3)$. At intermediate and high redshifts the volume probed by such a deep survey will contain several thousands of objects of interest $(N \sim 3500$, of which about 2000 screens possess at least $10 \mathrm{RMs}$ ). However, with the requirement of follow-up to obtain the magnetic field strength from the measured RM grid values, the number of objects, whose SZ imprint or X-ray emission can be detected by current or up-coming telescopes, drops to several hundred $(N \sim 300)$. Nevertheless, the deep survey sample extends to far higher redshifts than it is the case for the wide field one.
Thus, the wide and deep field RM SKA survey missions are actually complimentary and explore cosmic magnetism in LSS at different epochs.

\section{Conclusions}

We developed polarisation simulations of a high degree of realism within the SKADS project. The simulations have been used to make predictions about how instrument design affects polarisation studies and also utilised to make predictions for planned SKA survey missions. Furthermore, we have implemented and evaluated Bayesian data analysis methods in order to analyse spectro-polarimetric data and to obtain RMs from data of up-coming and future radio telescopes.

\section{References}

Alexander, P., Krause, M., Bolton, R., Riley, J.,\& Geisbuesch, J. 2007, PoS(MRU)068

Condon, J., Cotton, W.D., Greisen, E.W., Yin, Q.F., Perley, R.A., Taylor, G.B.,\& Broderick, J.J. 1998, AJ, 115, 1693 A\&A, 200, 58

Geisbuesch, J.,\& Alexander, P. 2009a, RMxAC, 36, 312

Geisbuesch, J.,\& Alexander, P. 2009b, submitted

Geisbuesch, J., Alexander, P., Krause, M.,\& Bolton, R. 2008 N. Cim. B, 122, 1253

Govoni, F., Murgia, M., Feretti, L., Giovannini, G., Dolag, K., Taylor, G. B. 2006, A\&A, 460, 425

Krause, M., Alexander P., Bolton R., Geisbuesch J., Green D. A.,\& Riley J. 2009 MNRAS, 400, 646

Mesa, D., Baccigalupi, C., et al. 2002, A\&A, 386, 463

Murgia, M., Govoni, F., Feretti, L., Giovannini, G., Dalacasa, D., Fanti, R., Taylor, G.,\& Dolag, K. 2004, A\&A, 424, 429

Schilizzi, R. T., et al., 2007, SKA Memo 100

Tucci, M., Martinez-Gonzalez E., Toffolatti L., GonzalezNuevo J.,\& de Zotti G. 2003, NewA Rev., 47, 1135

Wilman, R.J., Miller L., Jarvis M. J., Mauch T., Levrier F., Abdalla F. B., Rawlings S., Klckner H.-R., Obreschkow D., Olteanu D.,\& Young S. 2008, MNRAS, 388, 1335 